\title{
Work out optimization in the induced electromagnetic field calculation due to a lightning stroke
}

\section{J. A. Gutiérrez-Robles ${ }^{1}$, V. H. Ortiz-Muro ${ }^{2}$, O. Aguilar-Peña ${ }^{3}$, M. Á. Villalobos-Castro ${ }^{4}$, S. $^{-}$ A. Vargas-Sánchez ${ }^{5}$}

${ }^{1}$ Departamiento de Matemáticas, Universidad de Guadalajara, Av. Juárez No. 976, Colonia Centro, Guadalajara, Jalisco, México, C.P. 44100, Teléfono: +52 (33) 3134222.

${ }^{2}$ Departamiento de Mecánica Eléctrica, Universidad de Guadalajara, Av. Juárez No. 976, Colonia Centro, Guadalajara, Jalisco, México, C.P. 44100, Teléfono: +52 (33) 3134222.

3,4,5 Centro Nacional de Control de Energía - CENACE, Av. Vicente Guerrero No. 1234, Colonia Agua Blanca Industrial, Zapopan, Jalisco, México, C.P. 45235, Teléfono:52 (33) 36686442.

Email: jose.gutierrez@ cucei.udg.mx, victor.ortiz@cucei.udg.mx,oap255@hotmail.com,miguel.villalobos@cfe.gob.mx, sergio.vargas02@cfe.gob.mx.

Received: November $09^{\text {th }}, 2017$

Accepted: November, $20^{\text {th }}, 2017$

Published: December, $13^{\text {th }}, 2017$

Copyright (2016 by authors and Institute of Technology Galileo of Amazon (ITEGAM).This work is licensed under the Creative Commons Attribution International License (CC BY 4.0).

http://creativecommons.org/licenses/by/4.0/ (c) (i) (3) Open Acees:

\section{ABSTRACT}

In the electromagnetic field work out, due to indirect lightning discharge, the consumption of computational resources is essentially in the numerical integration of the Maxwell equations. These equations are solved in each discretized point for each time step because the work out of the field involves the inverse of the distance that's powered by 2, 3, 4 and 5; additionally its depends on the wave incident angle, which varies point by point, so it is impossible to interpolate, in a traditional way, intermediate data to optimize the calculus. It is proposed here the implementation of a parabolic space interpolation to reduce the computational needed resources; the compromise is the electromagnetic field calculation in the minimum time limiting the error to $0.2 \%$. It is looking for the optimum points, methodologically, to implement this interpolation by checking the results against the obtained ones without the omission of any point, so it is considered that this strategy is a good option in the electromagnetic field optimum calculation

Keywords: Electromagnetic field, lightning discharge, optimization.

\section{Optimización del cálculo de campo electromagnético inducido debido a una descarga atmosférica}

\section{RESUMEN}

En el cálculo del campo electromagnético debido a una descarga atmosférica, el consumo de recursos de cómputo es esencialmente en la integración numérica de las ecuaciones de maxwell, las cuales se integran en cada punto de discretización para cada paso de tiempo debido a que el cálculo del campo involucra el inverso de la distancia cuya potencia es $2,3,4$, y 5; adicionalmente depende del ángulo de incidencia de la onda, el cual varía punto a punto, por lo que no hay forma de interpolar de manera tradicional datos intermedios para optimizar dicho calculo. En este trabajo se propone una interpolación espacial parabólica que reduce los tiempo de computo sin introducir errores mayores a un $0.2 \%$. Se hace la búsqueda metodológica de los puntos óptimos para la implementación y se checan los resultados comparándolos con los que se obtienen del cálculo sin omitir ningún punto, por lo anterior se considera que esta estrategia es adecuada para el cálculo optimo del campo electromagnético.

Palabras-clave: Campo electromagnético, descarga atmosférica, optimización. 


\section{INTRODUCCIÓN}

Considere el diagrama de la Figura 1, ahí se muestra una línea de un kilómetro de longitud, de dos centímetros de radio, posicionada horizontalmente 10 metros sobre el piso. Se asume que el conductor es homogéneo y se supone que el piso también es homogéneo.

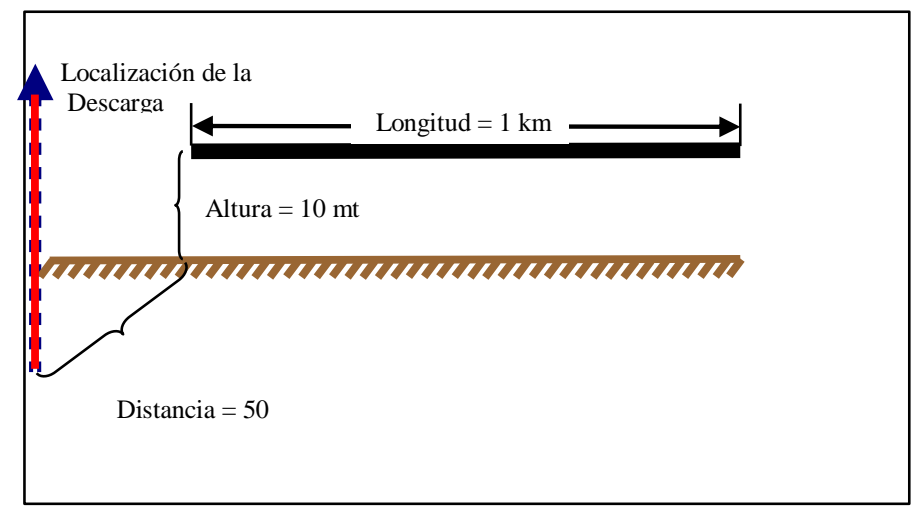

Figura 1: Diagrama utilizado para la simulación. Fuente: Autores, (2017).

Considerando que la tierra es un plano conductor perfecto, es posible deducir las ecuaciones de campo electromagnético de un dipolo vertical $d z^{\prime}$ a una altura $z^{\prime}$ a lo largo del canal de la descarga, resolviendo las ecuaciones de Maxwell en términos del Potencial con retardo como:

$$
\begin{aligned}
& \mathrm{d} E_{r}(r, \phi, z, t)=\frac{\mathrm{d} z^{\prime}}{4 \pi \varepsilon_{0}}\left\{\frac{3 r\left(z-z^{\prime}\right)}{R^{5}} \int_{0}^{\mathrm{t}} i\left(z^{\prime}, \tau-R / c\right) d \tau\right. \\
& \left.+\frac{3 r\left(z-z^{\prime}\right)}{c R^{4}} i\left(z^{\prime}, \tau-R / c\right)+\frac{r\left(z-z^{\prime}\right)}{c^{2} R^{3}} \frac{\partial i\left(z^{\prime}, \tau-R / c\right)}{\partial t}\right\} \\
& \mathrm{d} E_{z}(r, \phi, z, t)=\frac{\mathrm{d} z^{\prime}}{4 \pi \varepsilon_{0}}\left\{\frac{2\left(z-z^{\prime}\right)^{2}-r^{2}}{R^{5}} \int_{0}^{\mathrm{t}} i\left(z^{\prime}, \tau-R / c\right) d \tau\right. \\
& \left.+\frac{2\left(z-z^{\prime}\right)^{2}-r^{2}}{c R^{4}} i\left(z^{\prime}, \tau-R / c\right)+\frac{r^{2}}{c^{2} R^{3}} \frac{\partial i\left(z^{\prime}, \tau-R / c\right)}{\partial t}\right\} \\
& \mathrm{d} B_{\phi}(r, \phi, z, t)=\frac{\mu_{0} \mathrm{~d} z^{\prime}}{4 \pi}\left\{\begin{array}{c}
\frac{r}{R^{3}} i\left(z^{\prime}, \tau-R / c\right)+ \\
\left.\frac{r}{c R^{2}} \frac{\partial i\left(z^{\prime}, \tau-R / c\right)}{\partial t}\right\}
\end{array}\right.
\end{aligned}
$$

donde $i\left(z^{\prime}, t\right)$ es la corriente a lo largo del canal en $z^{\prime}, c$ es la velocidad de la luz, $\varepsilon_{0}$ y $\mu_{0}$ son la permitividad y la permeabilidad del aire respectivamente y $R=\sqrt{r^{2}+\left(z-z^{\prime}\right)^{2}}$.

El primer término de (1) y (2) es la componente de campo eléctrico estático en dirección de $r$ y $z$ respectivamente, el segundo término es el campo eléctrico inducido y el tercero es el campo eléctrico radiado. El primer término en (3) es el campo magnético inducido y el segundo es el campo magnético radiado. El campo total debido a una descarga atmosférica se obtiene de integrar las ecuaciones (1-3) a lo largo del canal de la descarga y su imagen; así se obtiene:

$$
\begin{aligned}
\mathrm{d} E_{r}(r, z, t)=\frac{1}{4 \pi \varepsilon_{0}}\left\{\int_{-\mathrm{H}}^{\mathrm{H}}\right. & \frac{3 r\left(z-z^{\prime}\right)}{R^{5}} \int_{0}^{\mathrm{t}} i\left(z^{\prime}, \tau-R / c\right) d \tau d z^{\prime} \\
& +\int_{-\mathrm{H}}^{\mathrm{H}} \frac{3 r\left(z-z^{\prime}\right)}{c R^{4}} i\left(z^{\prime}, \tau-R / c\right) d z^{\prime} \\
& \left.+\int_{-\mathrm{H}}^{\mathrm{H}} \frac{r\left(z-z^{\prime}\right)}{c^{2} R^{3}} \frac{\partial i\left(z^{\prime}, \tau-R / c\right)}{\partial t} d z^{\prime}\right\}
\end{aligned}
$$

$$
\begin{aligned}
& \begin{aligned}
\mathrm{d} E_{z}(r, z, t)=\frac{1}{4 \pi \varepsilon_{0}}\left\{\int_{-\mathrm{H}}^{\mathrm{H}} \frac{2\left(z-z^{\prime}\right)^{2}-r^{2}}{R^{5}} \int_{0}^{\mathrm{t}} i\left(z^{\prime}, \tau-R / c\right) d \tau d z^{\prime}\right. \\
\\
+\int_{-\mathrm{H}}^{\mathrm{H}} \frac{2\left(z-z^{\prime}\right)^{2}-r^{2}}{c R^{4}} i\left(z^{\prime}, \tau-R / c\right) d z^{\prime}
\end{aligned} \\
& \left.\quad+\int_{-\mathrm{H}}^{\mathrm{H}} \frac{r^{2}}{c^{2} R^{3}} \frac{\partial i\left(z^{\prime}, \tau-R / c\right)}{\partial t} d z^{\prime}\right\} \\
& \begin{aligned}
\mathrm{d} B_{\phi}(r, \phi, z, t)=\frac{\mu_{0}}{4 \pi}\left\{\int_{-\mathrm{H}}^{\mathrm{H}} \frac{r}{R^{3}} i\left(z^{\prime}, \tau-R / c\right) d z^{\prime}\right. \\
+
\end{aligned} \\
& \text { II. ACOPLAMIENTO ELECTROMAGNÉTICO ENTRE EL } \\
& \text { CAMPO Y LOS CONDUCTORES DE LA LÍNEA }
\end{aligned}
$$

\section{ACOPLAMIENTO ELECTROMAGNÉTICO ENTRE EL CAMPO Y LOS CONDUCTORES DE LA LÍNEA}

Para calcular el campo electromagnético es necesario conocer la distribución de la corriente en espacio-tiempo a lo largo del canal de la descarga $i\left(z^{\prime}, t\right)$. De acuerdo al modelo modificado de línea de transmisión, la distribución tiempoespacio de la corriente se expresa como:

$$
\begin{array}{cc}
i\left(z^{\prime}, t\right)=i\left(0, t-z^{\prime} / v\right) e^{\left(-z^{\prime} / \lambda\right)} & z^{\prime} \leq v t \\
i\left(z^{\prime}, t\right)=0 & z^{\prime}>v t
\end{array}
$$

donde $v$ es la velocidad del canal de retorno y $\lambda$ es el factor de atenuación de la corriente. Esta atenuación no representa pérdidas a lo largo del canal de la descarga pero se propone para tomar en cuenta el efecto de la carga debido al canal principal y las descargas de retorno sucesivas.

Una aproximación rigurosa en el cálculo de la corriente y el voltaje inducidos en una línea de distribución aérea debido a una descarga indirecta se realiza con la teoría de antenas. Una simplificación se realiza utilizando una aproximación quasiestática, la cual describe el acoplamiento entre el campo electromagnético y los conductores de una línea aérea con parámetros concentrados. Esta aproximación es válida solo si la dimensión de la línea es diez veces menor que la longitud de onda más pequeña involucrada en el fenómeno. Por lo tanto esta aproximación no es aplicable para líneas de transmisión aéreas, iluminadas por una descarga atmosférica, de varios kilómetros de longitud.

Por otro lado, la aproximación dada por la teoría de líneas ofrece un balance adecuado, esta se basa en dos hipótesis:

1. La dimensión transversal de la línea es menor que la longitud de onda significativa más pequeña del campo electromagnético incidente.

2. La respuesta de la línea es quasi-transversal electromagnética.

Las ecuaciones de acoplamiento para una línea multiconductora homogénea sin perdidas, que está posicionada horizontalmente respecto a tierra, e iluminada por un campo electromagnético debido a una descarga atmosférica son:

$$
\begin{aligned}
& \frac{\partial u^{s}(x, t)}{\partial x}+\mathbf{L}^{\prime} \frac{\partial i(x, t)}{\partial t}=\mathbf{E}_{x}^{i}(x, h, t) \\
& \frac{\partial i(x, t)}{\partial x}+\mathbf{C}^{\prime} \frac{\partial u^{s}(x, t)}{\partial t}=\mathbf{0}
\end{aligned}
$$

donde $\mathbf{E}_{x}^{i}(x, h, t)$ es el vector de la componente horizontal de 
campo eléctrico incidente a lo largo del eje $x$ a una altura de conductor $h_{i}$, el subíndice denota un conductor de la línea multiconductora, $i(x, t)$ es el vector de corrientes inducidas de la línea, $\mathbf{L}^{\prime}$ y $\mathbf{C}^{\prime}$ son las matrices de inductancia y capacitancia por unidad de longitud respectivamente, $u^{s}(x, t)$ es el vector de voltaje estático.

El voltaje estático se relaciona con el voltaje total por:

$$
u(x, t)=u^{i}(x, t)+u^{s}(x, t)
$$

donde el voltaje incidente es:

$$
u^{i}(x, t)=-\int_{0}^{h} \mathbf{E}_{z}^{i}(x, z, t) d z
$$

aquí $\mathbf{E}_{z}^{i}(x, z, t)$ es el campo eléctrico vertical inducido el cuál se puede considerar invariante respecto a la altura en el intervalo de interés $0<z<h_{i}$.

\section{CALCULO DEL CAMPO ELECTROMAGNÉTICO PARA UNA LÍNEA DE 1 KM DE LONGITUD}

El cálculo numérico del campo eléctrico horizontal a lo largo del eje $\mathrm{x}$ se implementa utilizando un $\Delta t=1 e^{-8} \mathrm{seg}$ segundos y $\Delta x=10 \mathrm{mt}$ como se muestra en la Figura 2.

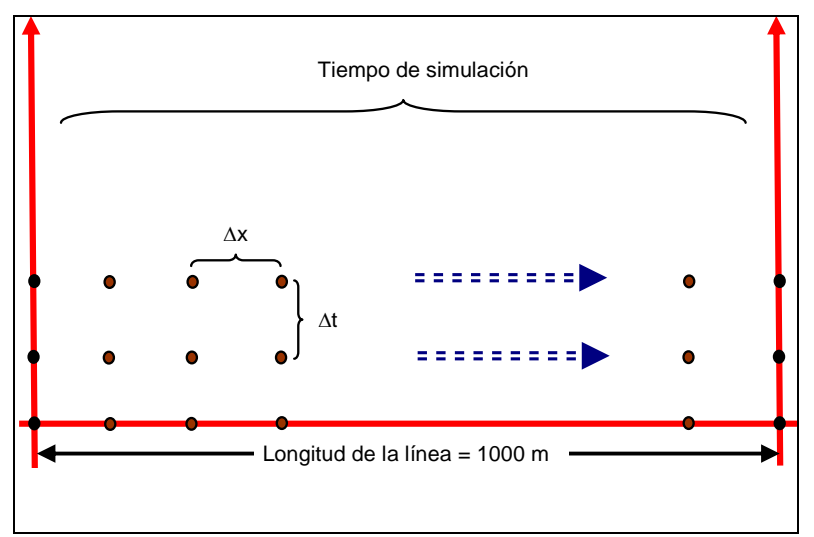

Figura 2: Diagrama de la discretizacion de la línea.

Fuente: Autores, (2017).

Con los datos de la Figura 1 y 2, se hace el cálculo de campo electromagnético debido a una descarga atmosférica con una corriente dada por la función de Heidler, una en forma triangular 2/25 microsegundos y una en forma trapezoidal con un tiempo de elevación de 2 microsegundos. Se hicieron 100 simulaciones en MatLab y se promediaron los tiempos de ejecución; la Tabla 1 muestra los tiempos promedios para cada forma de onda de la corriente con un tiempo de simulación de 12 microsegundos.

Tabla 1: Tiempos promedio de simulación para los diferentes tipos de corrientes de descarga atmosférica.

\begin{tabular}{|l|c|}
\hline \multicolumn{1}{|c|}{$\begin{array}{c}\text { Tipo de corriente de la } \\
\text { descarga }\end{array}$} & $\begin{array}{c}\text { Tiempo promedio en 100 } \\
\text { ejecuciones }\end{array}$ \\
\hline Heidler & 334.8600 Segundos \\
\hline Triangular & 300.3280 Segundos \\
\hline Trapezoidal & 186.4850 Segundos \\
\hline
\end{tabular}

Fuente: Autores, (2017).
Analizando las ecuaciones (4), (5) y (6) se puede fácilmente deducir que para calcular un punto en espacio y tiempo, es necesario hacer 8 integrales numéricamente. Es aquí donde se consume el tiempo de cómputo.

Para acelerar este proceso, una práctica ya muy conocida es implementar ventanas temporales, es decir, en una primer ventana de tiempo se calcula el campo electromagnético muestra a muestra; en una segunda etapa se calcula el campo cada ciertas muestras y el resto se estiman por interpolación lineal. Lo anterior se muestra gráficamente en la Figura 3.

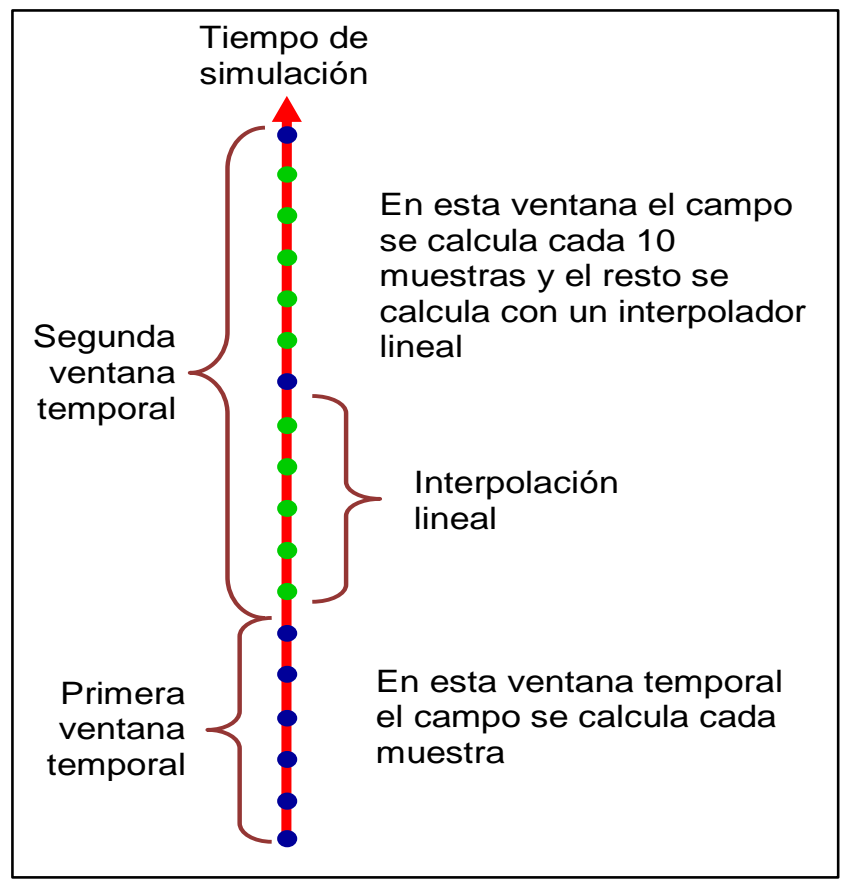

Figura 3: Esquema de la interpolación lineal en tiempo. Fuente: Autores, (2017).

Utilizando el esquema anterior con una primera ventana temporal en el intervalo de 0 a $2 \square \mathrm{s}$ y una segunda ventana temporal de 2 a $12 \square \mathrm{s}$, con un espaciamiento de 10 muestras entre cálculos, la Tabla 2 resume los tiempos promedios de simulación en 100 ejecuciones.

Tabla 2: Tiempo promedio en la simulación utilizando el esquema de dos ventanas temporales e interpolación lineal en tiempo.

\begin{tabular}{|l|c|}
\hline \multicolumn{1}{|c|}{$\begin{array}{c}\text { Tipo de corriente de la } \\
\text { descarga }\end{array}$} & $\begin{array}{c}\text { Tiempo promedio en 100 } \\
\text { ejecuciones }\end{array}$ \\
\hline Heidler & 73.8600 Segundos \\
\hline Triangular & 51.1250 Segundos \\
\hline Trapezoidal & 38.7970 Segundos \\
\hline
\end{tabular}

Fuente: Autores, (2017).

Una aceleración adicional se logra implementando una interpolación espacial; debido a que en espacio se tiene un comportamiento no lineal, no se puede elegir de manera simple cual punto se puede interpolar y cual no. Como primera aproximación se eligen de manera simple los puntos pivote como se muestra en la Figura 4b, es decir, el problema original se muestra en la Figura 4a donde se calculan todos los puntos y se cambia por el de la Figura $4 \mathrm{~b}$ donde los puntos indicados se calculan por integración numérica y el resto por interpolación. 

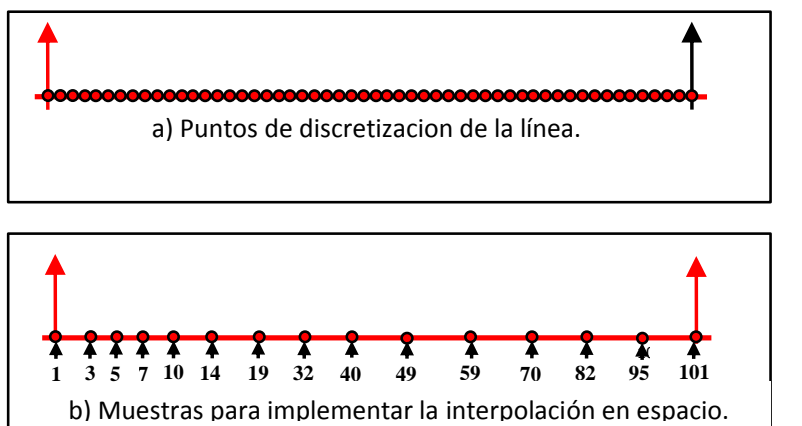

Figura 4: a) Total de puntos, b) Puntos elegidos.

Fuente: Autores, (2017).

La Tabla 3 resume los resultados que se obtienen cuando se utilizan los puntos de la figura anterior como base de la interpolación en espacio.

Tabla 3: Tiempo promedio en la simulación utilizando el esquema de dos ventanas temporales e interpolación parabólica en espacio.

\begin{tabular}{|l|c|}
\hline \multicolumn{1}{|c|}{$\begin{array}{c}\text { Tipo de corriente de la } \\
\text { descarga }\end{array}$} & $\begin{array}{c}\text { Tiempo promedio en 100 } \\
\text { ejecuciones }\end{array}$ \\
\hline Heidler & 13.686000 \\
\hline Triangular & 8.906000 \\
\hline Trapezoidal & 7.090150 \\
\hline
\end{tabular}

Fuente: Autores, (2017).

Se integran todos los puntos en espacio y tiempo, se toman los tiempos de simulación como referencia para hacer comparaciones de ahorro en recursos de cómputo. La Tabla 4 muestra los resultados, en por ciento de tiempo utilizado, implementando las dos técnicas para reducir tiempo de computo. En una columna se toma el tiempo de la simulación completa como $100 \%$, en otra columna se toma el por ciento de este tiempo que se toma si se implementa la interpolación lineal y finalmente el tiempo que se toma la simulación si se implementa adicionalmente la interpolación parabólica.

Tabla 4: Tiempo de simulación promedio para diferentes tipos de formas de onda, implementando las metodologías propuestas.

\begin{tabular}{|l|c|c|c|}
\hline $\begin{array}{c}\text { Tipo de } \\
\text { corriente de la } \\
\text { descarga }\end{array}$ & Completo & $\begin{array}{c}\text { Interpolación } \\
\text { lineal en } \\
\text { tiempo }\end{array}$ & $\begin{array}{c}\text { Adicionalmente } \\
\text { interpolación } \\
\text { parabólica }\end{array}$ \\
\hline Heidler & $100 \%$ & $22.057 \%$ & $4.087 \%$ \\
\hline Triangular & $100 \%$ & $17.023 \%$ & $2.965 \%$ \\
\hline Trapezoidal & $100 \%$ & $20.804 \%$ & $3.802 \%$ \\
\hline
\end{tabular}

Fuente: Autores, (2017).

\section{ANÁLISIS DE RESULTADOS}

Se calcula el campo eléctrico vertical y horizontal incidente en cada punto de discretización de la línea como se muestra en la Figura 4a. Para evaluar el error que se introduce por la interpolación lineal, se hace el cálculo implementando las dos ventanas temporales, los resultados de ambas simulaciones se comparan en forma directa, para este caso especifico estos resultados se muestran en las figuras 5 y 6 ; para las simulaciones se utiliza una función para la descarga atmosférica de $30 \mathrm{kA}$ y una forma trapezoidal $2 / 50 \mu \mathrm{s}$.

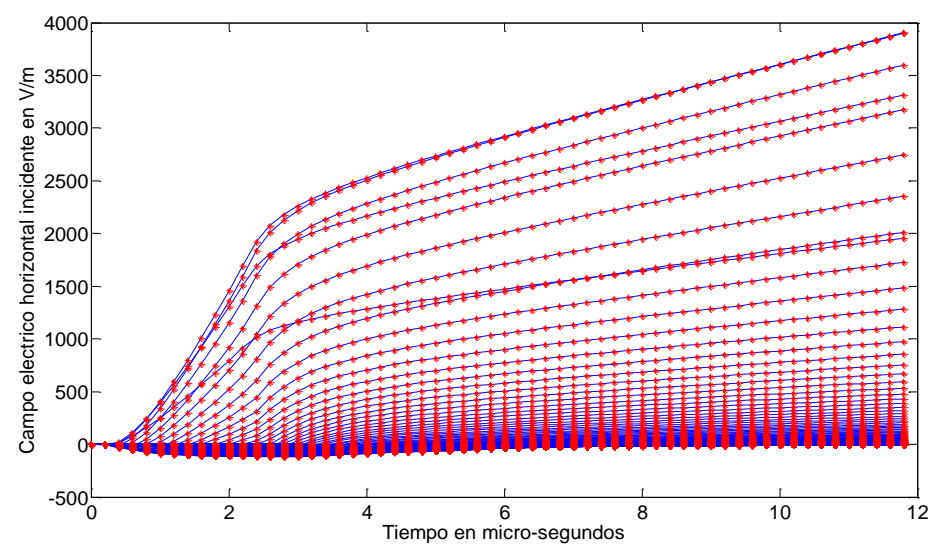

Figura 5: Campo eléctrico horizontal incidente, sin ninguna simplificación y utilizando las dos ventanas temporales.

Fuente: Autores, (2017).

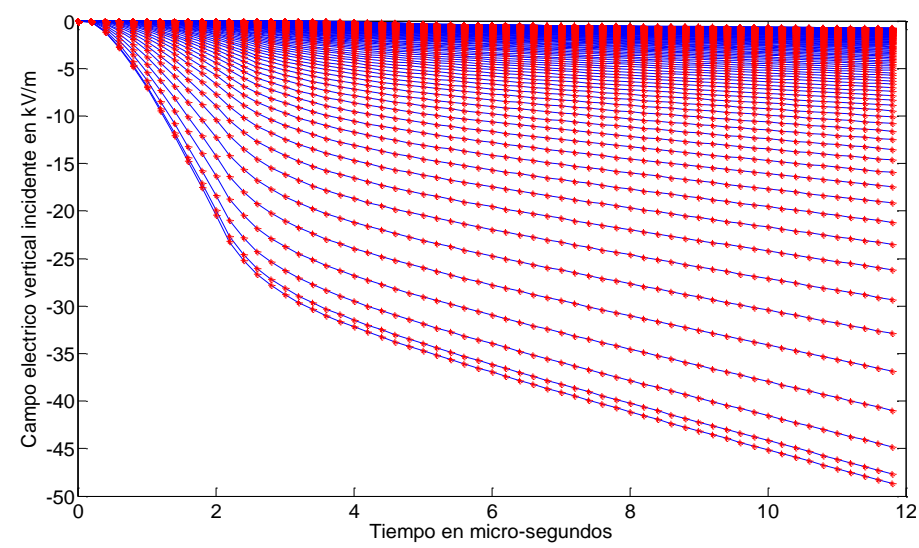

Figura 6. Campo eléctrico vertical incidente, sin ninguna simplificación y utilizando las dos ventanas temporales.

Fuente: Autores, (2017).

El error porcentual que incorpora el uso de la interpolación lineal se calcula con la siguiente fórmula:

$$
\text { Error }=\frac{\mathbf{E}_{h}^{i}(\text { base })-\mathbf{E}_{h}^{i}(t w)}{\max \left(\mathbf{E}_{h}^{i}(\text { base })\right)} \times 100 \%
$$

donde $\mathbf{E}_{h}^{i}($ base $)$ es el campo eléctrico horizontal/vertical incidente sin ninguna simplificación y $\mathbf{E}_{h}^{i}(t w)$ es el campo eléctrico horizontal/vertical incidente utilizando las dos ventanas temporales. Las figuras 7 y 8 muestran los errores para cada caso en forma respectiva.

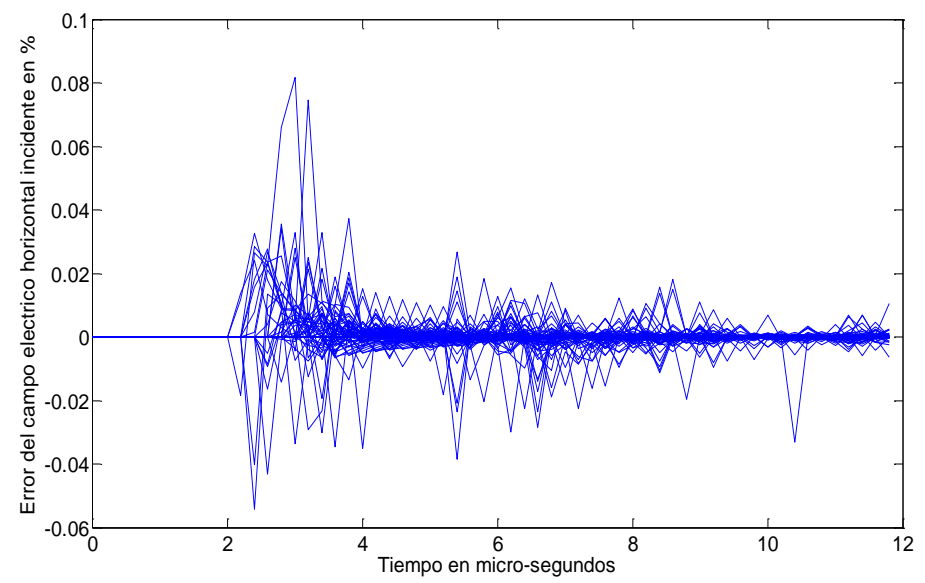

Figura 7. Error del campo eléctrico vertical incidente.

Fuente: Autores, (2017). 


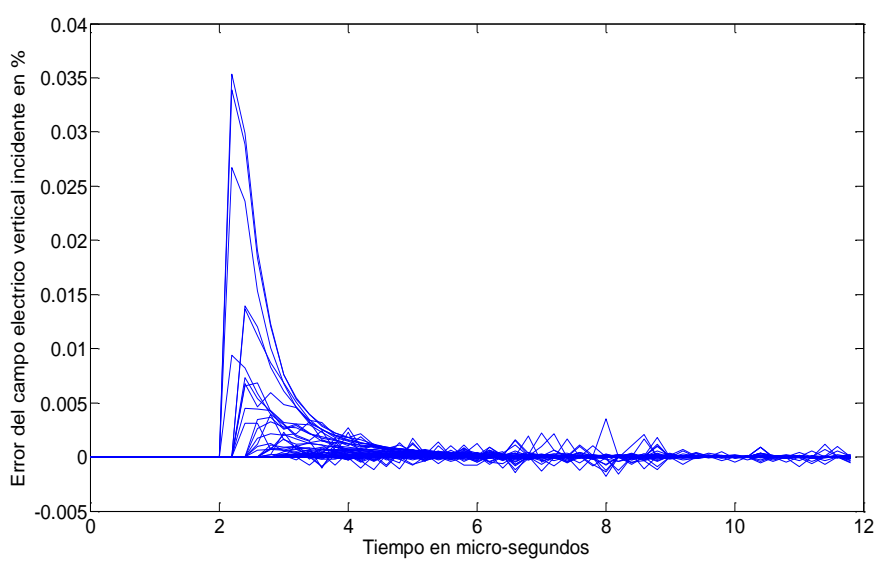

Figura 8: Error del Campo eléctrico vertical incidente.

Fuente: Autores, (2017).

El paso siguiente es la implementación de la interpolación parabólica en espacio, con los puntos base que se muestran en la Figura 4b. Los resultados que se obtienen se muestran en la figura 9 y 10. En estas figuras en negro se muestran las curvas utilizadas para interpolar y en línea punteada las interpoladas.

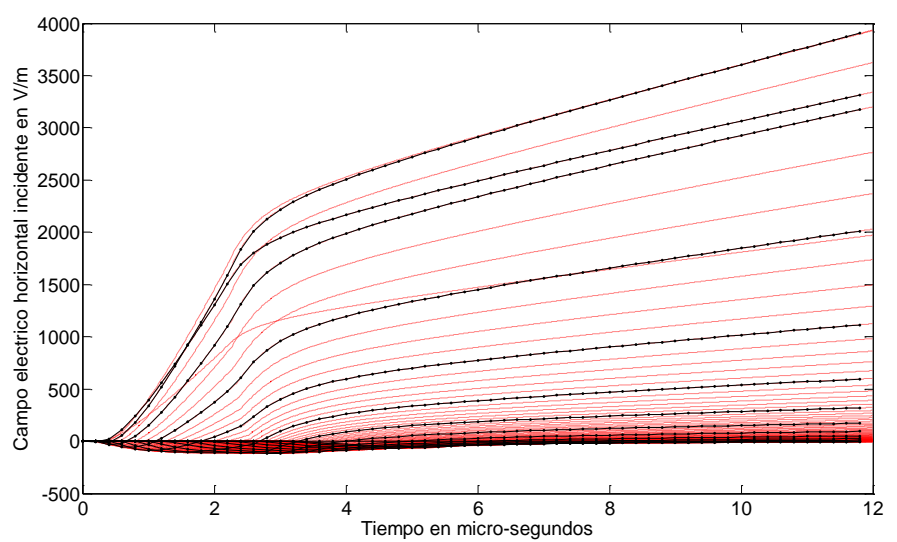

Figura 9: Campo eléctrico horizontal incidente.

Fuente: Autores, (2017).

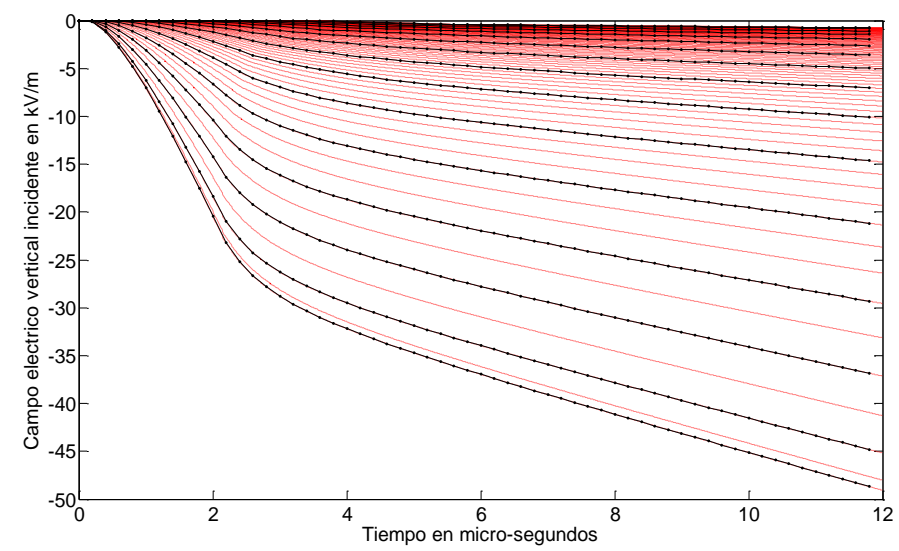

Figura 10: Campo eléctrico vertical incidente.

Fuente: Autores, (2017).

En este caso en específico las curvas base se calculan sin ninguna simplificación, es decir, el error que introduce el uso de la interpolación parabólica en espacio es adicional al que se introduce por el uso de la interpolación lineal. La suma de ambos errores será el error total en el cálculo de campo eléctrico horizontal/vertical incidente. Las figuras 11 y 12 muestran el error que se introduce por el uso de la interpolación en espacio.

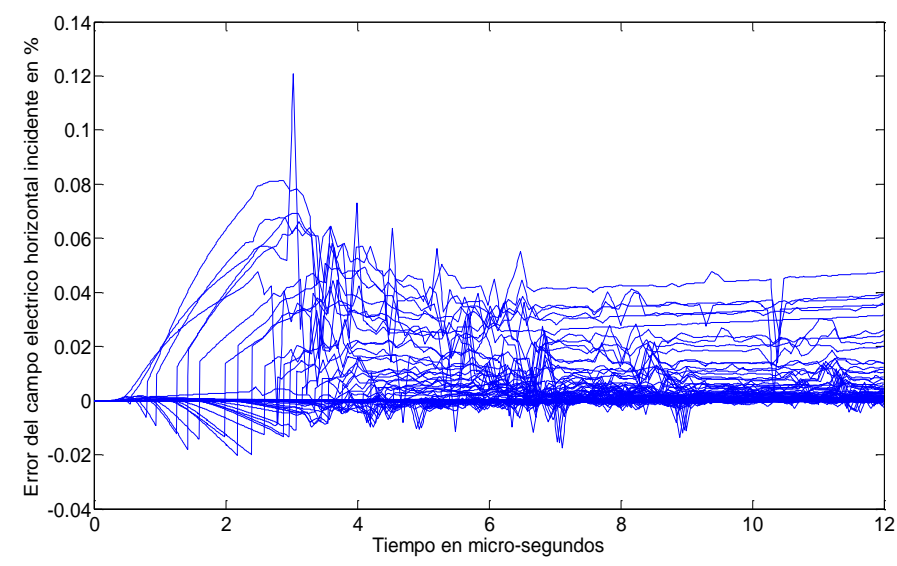

Figura 11: Error del campo eléctrico horizontal incidente. Fuente: Autores, (2017).

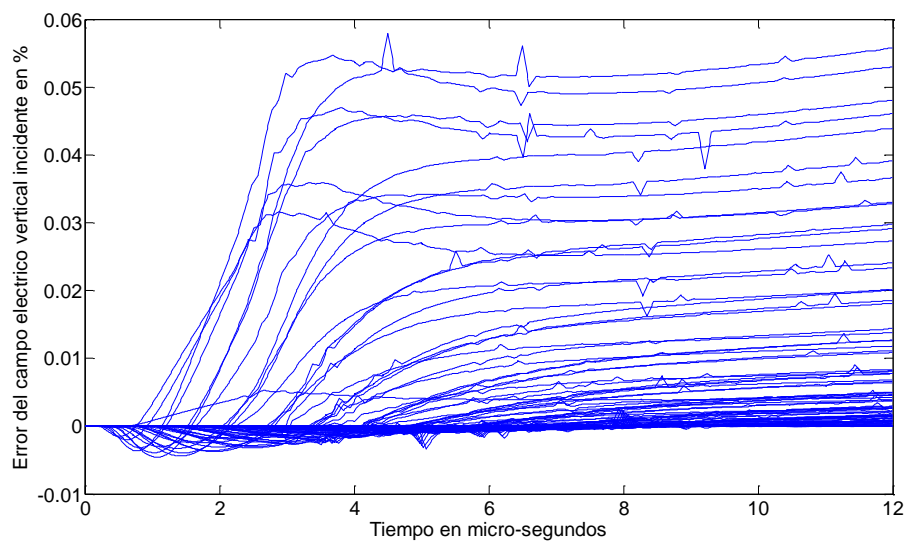

Figura 12: Error del campo eléctrico vertical incidente Fuente: Autores, (2017).

\section{OPTIMIZACIÓN EN EL CÁLCULO DEL CAMPO ELECTROMAGNÉTICO}

El ejemplo que se menciona anteriormente es ambiguo, porque los puntos que se tomaran para realizar el cálculo del campo electromagnético espacial se tomaron de forma arbitraria. El ejemplo que se utilizara para crear la Heurística para encontrar los puntos de interpolación espacial respecto a un margen de error no mayor a $0.2 \%$, es el siguiente; se tiene una línea de $3 \mathrm{Km}$ de longitud, de $2 \mathrm{~cm}$ de radio y $10 \mathrm{~m}$ de altura respecto al piso. Se tiene una descarga atmosférica 30 metros de uno de los extremos, perpendicular a la línea. La distancia de la descarga atmosférica aumenta de 10 metros en 10 metros hasta los 500 metros. El esquema grafico de este ejemplo se muestra en la Figura 13.

La metodología para determinar los puntos óptimos de interpolación para una distancia específica se desarrolla de la siguiente manera:

1.- Se toma una línea de $3 \mathrm{~km}$ de longitud, la razón detrás de esta elección es que a una distancia mayor la inducción es muy baja y no es necesario por tanto hacer el análisis para distancias mayores.

2.- Se discretiza la línea; en este caso la distancia espacial entre muestras es de 10 metros, con un paso en tiempo de 10 nanosegundos.

3.- Se toma una descarga atmosférica a cierta distancia perpendicular a uno de los extremos de la línea, la primera se toma a 30 metros porque a distancias menores es prácticamente como tener una descarga directa. 


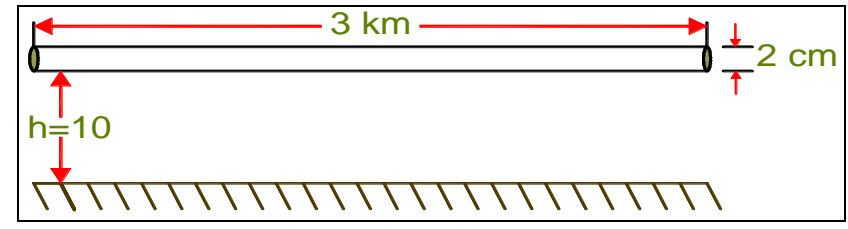

a)

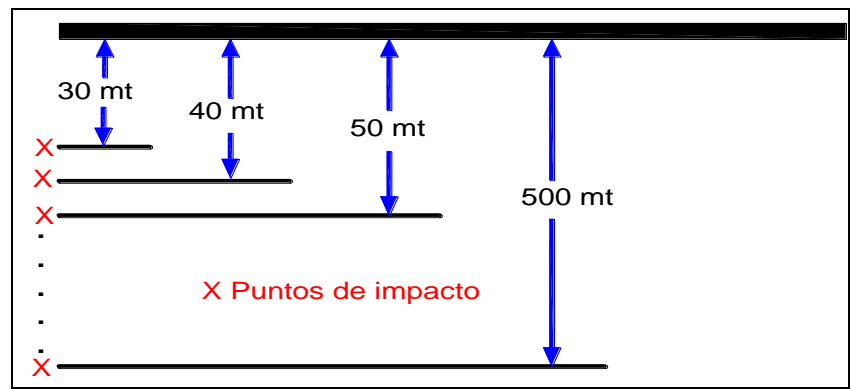

b)

Figura 13: a) Vista frontal y b) vista aérea y puntos de impacto en una línea de distribución de $3 \mathrm{~km}$ de longitud.

Fuente: Autores, (2017).

4.- Se calcula el campo electromagnético en todos los puntos para cada paso de tiempo, este cálculo sirve como punto de referencia para el análisis de error.

5.- Se implemente la interpolación lineal en tiempo; es decir, se calcula muestra a muestra de 0 a 2 microsegundos, y de 2 microsegundos a 12 microsegundos se calcula cada 10 muestras. Las muestras intermedias se calculan por interpolación lineal.

6.- Se hace el cálculo de error de la implementación respecto al resultado exacto para verificar que no sobrepase el $0.2 \%$ de error determinado como referencia.

7.- Para la implementación de la interpolación espacial es necesario tener los puntos extremos como referencia, así la búsqueda de los demás puntos óptimos para la implementación procede de la siguiente manera:

a) En forma sistemática se omite el cálculo del punto dos en adelante.

b) Cada que se omite un punto, los datos de los costados se toman como base de la interpolación, esto se esquematiza en la Figura 14.

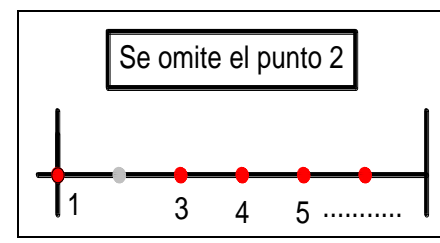

a)

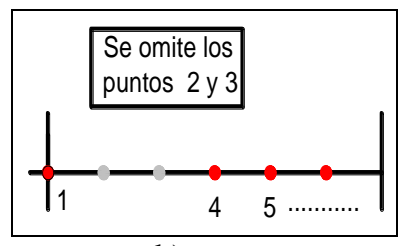

b)
Figura 14: Desarrollo de la obtención de puntos óptimos a) Se omite solo el punto 2, b) Se omiten los puntos 2 y 3 .

Fuente: Autores, (2017).

c) En el primer caso se toman los puntos 1 y 3 para calcular por interpolación el punto 2 para todo t. La curva 2 resultante de la interpolación se compara con su correspondiente resultado del cálculo directo determinando el error. Si este se encuentra por abajo del $0.2 \%$, este punto es susceptible de ser interpolado. d) Se procede a omitir también el punto 3; así los puntos 1 y 4 sirven de base para interpolar los puntos 2 y 3 . Se comparan ambos con sus correspondientes y si el error calculado es menor que el $0.2 \%$, el punto 3 también susceptible de ser interpolado.

e) Se continua el proceso hasta encontrar un punto con un error calculado mayor a $0.2 \%$, en este caso este punto que no puede ser interpolado se toma como base para la interpolación y se procede a omitir el siguiente punto por la derecha.

f) Se procede en forma metódica con este proceso hasta encontrar todos los puntos que no pueden ser interpolados y que por supuesto son los puntos base para la implementación de la interpolación parabólica espacial.

8).- La distancia de la descarga a la línea se aumenta en 10 metros y se realiza nuevamente el proceso antes descrito.

Aplicando este proceso al ejemplo descrito en la Figura 1, los puntos ideales para la implementación de la interpolación parabólica se muestran en la Figura 15.

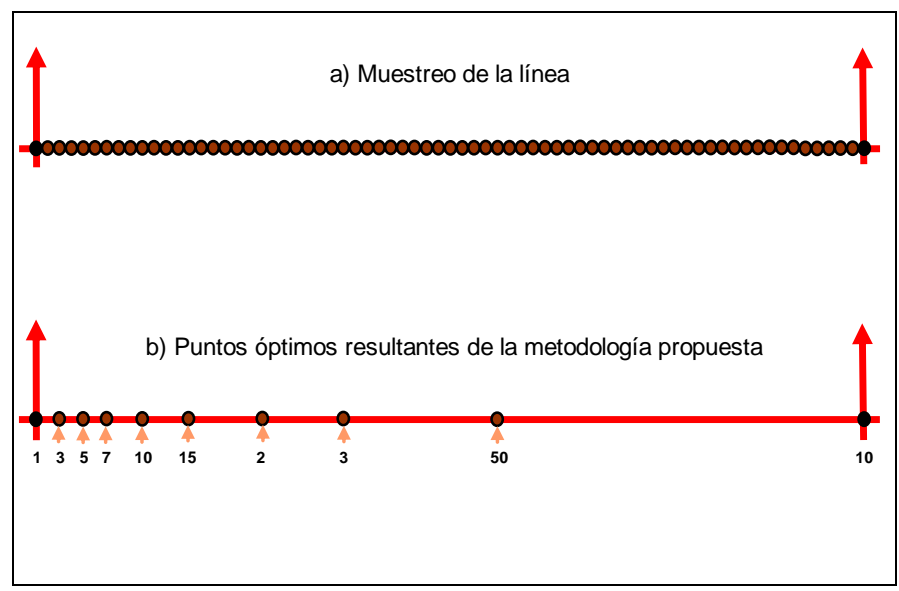

Figura 15: Puntos óptimos resultado de aplicar el proceso de selección.

Fuente: Autores, (2017).

La Tabla 5 muestra el resumen de resultados utilizando los puntos óptimos, mostrados en la figura 15, para implementar la interpolación.

Tabla 5: Tiempo promedio de simulación y porciento de tiempo utilizado para el cálculo del campo electromagnético utilizando las dos ventanas temporales y la interpolación parabólica en espacio.

\begin{tabular}{|l|c|c|}
\hline $\begin{array}{c}\text { Tipo de corriente } \\
\text { de la descarga }\end{array}$ & $\begin{array}{c}\text { Tiempo promedio } \\
\text { en } 100 \text { ejecuciones }\end{array}$ & $\begin{array}{c}\text { \% de tiempo } \\
\text { de ejecucion }\end{array}$ \\
\hline Heidler & 9.4356 & 2.8178 \\
\hline Triangular & 6.2617 & 2.0850 \\
\hline Trapezoidal & 5.0127 & 2.6880 \\
\hline
\end{tabular}

Fuente: Autores, (2017).

Las figuras 16 y 17 muestran el campo eléctrico horizontal/vertical incidente, respectivamente. La línea negra continua corresponde a las formas de onda calculadas por integración numérica y las líneas punteadas corresponden a las formas de onda interpoladas. Por otro lado las figuras 18 y 19 muestran los errores en el cálculo, en cada caso el error no sobrepasa el $0.2 \%$. 


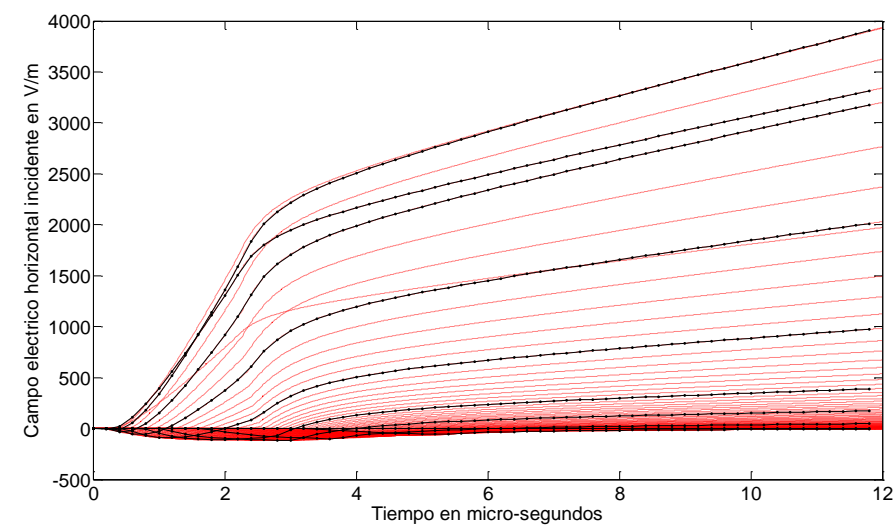

Figura 16: Campo eléctrico horizontal incidente.

Fuente: Autores, (2017).

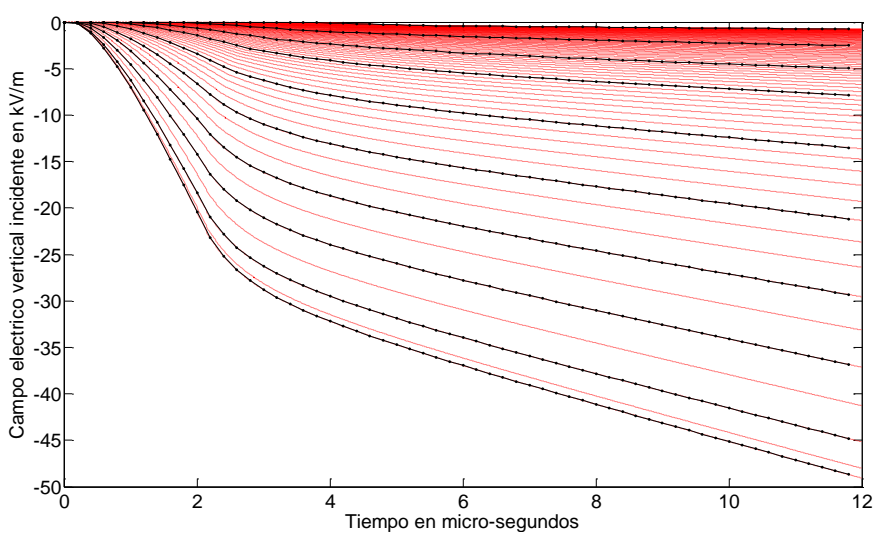

Figura 17: Campo eléctrico vertical incidente.

Fuente: Autores, (2017).

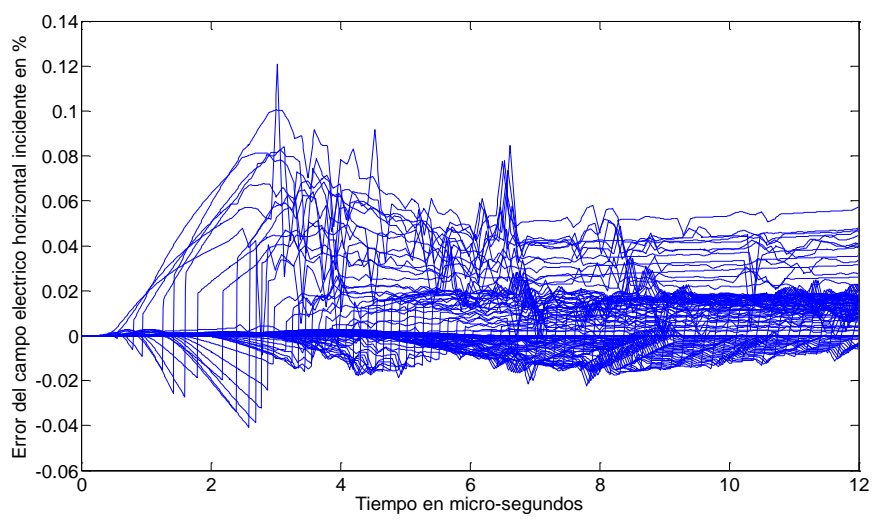

Figura 18: Error del campo eléctrico horizontal incidente. Fuente: Autores, (2017).

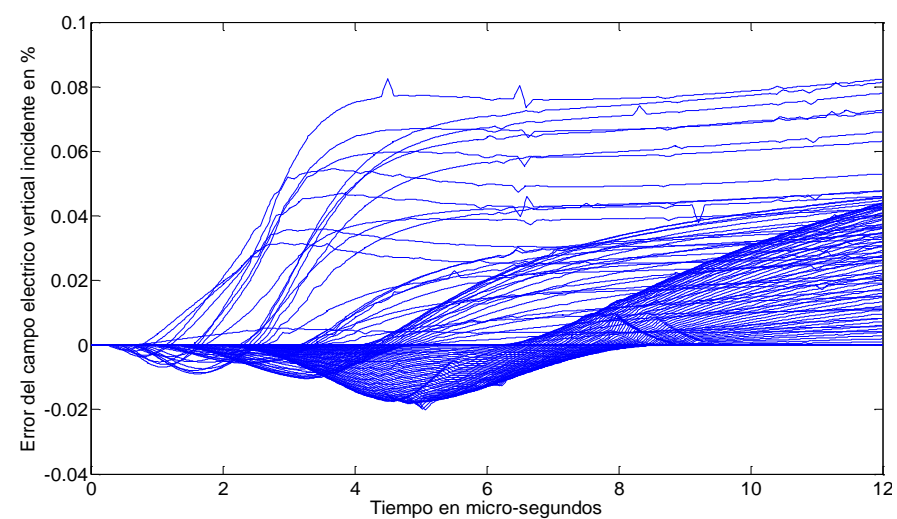

Figura 19: Error del campo eléctrico vertical incidente.

Fuente: Autores, (2017).

\section{CONCLUSIONES}

La interpolación lineal reduce substancialmente los tiempos de ejecución en el cálculo del campo electromagnético debido a una descarga atmosférica, el precio de esta reducción es un error incorporado de $0.1 \%$. Haciendo un compromiso entre reducción de tiempo de ejecución y error incorporado, la interpolación lineal en tiempo es el camino adecuado y correcto para reducir tiempos de ejecución.

Con la misma filosofía se encontró que la interpolación parabólica en espacio es tan buena como la interpolación lineal en tiempo. El error adicional incorporado por la nueva implementación es alrededor de $0.14 \%$, así la adopción de esta metodología es adecuada en el cálculo del campo electromagnético horizontal/vertical incidente. El uso combinado de ambas metodologías en forma simultánea reduce enormemente el tiempo de ejecución.

\section{REFERENCIAS}

[1] M. A. Uman, D. K. McLain, E. P. Krider, "The electromagnetic radiation from a finite antenna", Am. Journal of Physics, Vol. 43, pp. 33-38, 1975.

[2] M. J. Master, M. A. Uman, "Transient electric and magnetic fields associated with establishing a finite electrostatic dipole", Am. Journal of Physics, Vol. 51, pp. 118-126, 1983.

[3] M. Rubinstein, M. A. Uman, "Methods for calculating the electromagnetic fields from a known source distribution: application to lightning", IEEE Transactions on Electromagnetic Compatibility, Vol. EMC-31, No.2, pp. 183-189, May 1989.

[4] C. A. Nucci, C. Mazzetti, F. Rachidi, M. Ianoz, "On lightning return stroke models for LEMP calculation”, Proceeding $19^{\text {th }}$ International Conference on Lightning protection, Graz, April, 1990.

[5] F. M. Tesche, "Comparison of the transmission line and scattering models for computing the HEMP response of overhead cables", IEEE Transactions on Electromagnetic Compatibility, Vol. 34, No. 2, May 1992.

[6] S. Rusk, "Induced lightning overvoltages on power transmission lines with special reference to the overvoltages protection of low voltage networks", Transactions of the Royal Institute of Technology, Stockholm, No. 120, 1958.

[7] J. A. Gutiérrez, "Analysis of electromagnetic transients due to lighting in transmission towers and distribution lines", PhD Thesis, Cinvestav-Unidad Guadalajara, 2002. 